\title{
Efectos de la cuarentena y las sesiones virtuales en tiempos del COVID-19
}

\section{Effects of quarantine and virtual sessions in times of COVID-19}

\author{
Oriana M. Chacón-Lizarazo ${ }^{\mathrm{a}}$, Sophia Esquivel Núñez \\ a* Psicóloga, 0000-0003-0292-9713, oriiana9312@gmail.com, Centro de Investigación Barí Ixta (CIBI), Cúcuta, Colombia \\ bagister en Psicología Organizacional, 0000-0001-7425-2364, sophia.esquivel@solucionescreativas.net, Universidad Nacional, \\ Costa Rica
}

Forma de citar: O.M Chacón-Lizarazo, S. Esquivel-Núñez, Efectos de la cuarentena y las sesiones virtuales en tiempos del COVID-19. Eco Matemático, 11 (1), 31-39

Recibido: 5 de diciembre de 2019

Aceptado: 30 de diciembre de 2019

\section{Palabras clave}

COVID-19, terapias virtuales, salud mental.

*Autor para correspondencia oriiana9312@gmail.com

Resumen: Debido a la pandemia generada por el COVID-19, países de todo el mundo entraron en cuarentena total, modificando la cotidianidad de las personas drásticamente, una de las poblaciones más afectadas por los cambios son los niños, muchos de ellos con diversidad funcional y trastornos del desarrollo debieron parar sus procesos terapéuticos, es así como el articulo hace un intento en ahondar por los efectos de la cuarentena en dicha población infantil, así mismo, establecer los impactos generados por la virtualidad que ofrecen las sesiones terapéuticas desde esta modalidad. Es por ello que, a través del diseño de un cuestionario dividido en dos momentos, se logró concluir que dentro de los efectos colaterales del covid-19 por la cuarentena, se vieron afectadas en los niños las áreas emocionales, de comportamiento y el sueño, así mismo, el inicio de sesiones virtuales logró impactar de forma positiva, aportando no solo al objetivo propio de cada proceso de intervención sino también a la salud mental de la dinámica familiar. 


\section{Keywords}

COVID-19, virtual therapies, mental health.

\section{Introducción}

\section{Pandemia: COVID-19 en el 2020}

El año 2019 ha representado una gran cantidad de cambios y retos a nivel mundial, puesto que actualmente se vive la pandemia del Coronavirus, una situación que no estaba contemplada, pero que cada país, de acuerdo a sus recursos han intentado afrontar de la mejor manera posible. Aun así, es importante mencionar que el término pandemia no es desconocido, a lo largo de la historia, son muchas las pandemias que el mundo ha enfrenado, las últimas presentadas fueron la del Ébola que osciló entre 2014 y 2016, y anterior a ella la Gripe Porcina manifestada entre 2009 y 2010 (Tomasi, 2019), sin embargo, el COVID-19 es la primera del siglo XXI que llevó a varios países en Latinoamérica como Colombia y Costa Rica a un estado de cuarentena por más de dos meses.

Lo anterior ha generado cambios drásticos en el estilo de vida, puesto que alteró las rutinas familiares, sociales, laborales y académicas para que aquellos que estudian, llevando a tomar medidas alternativas donde han surgido términos como "teletrabajo", "clases virtuales" y "telemedicina", siendo la tecnología la mayor aliada en la actualidad, logrando facilitar gran parte de las responsabilidades y deberes. 
Queda claro que los cambios han recaído en todos los sectores y uno de los que se ha visto más afectado es la población infantil, entiendo aquí, que dentro de las rutinas y deberes cotidianos de muchos niños estaba no solo el colegio, dado que aquellos con diferentes diagnósticos asistían de manera constante a procesos terapéuticos personalizados y grupales. De acuerdo a ello, para los deberes académicos se realizaron adaptaciones como las clases virtuales, pero para dichas terapias las modificaciones aún están en el abanico de opciones, en primera instancia porque no son de carácter urgente y segundo, porque al ser terapias personalizadas requieren de adaptaciones concretas en los objetivos establecidos para cada una de las intervenciones.

Entendiendo el contexto y los cambios que se han generado en tan poco tiempo, algunos entes de salud han implementado "sesiones virtuales", con la intención de lograr una continuidad en dichos procesos terapéuticos que llevaban cada uno de los niños con diversidad funcional; de ahí que se cuestiona la probabilidad del impacto positivo frente a las sesiones virtuales en niños con diferentes trastornos del desarrollo, indagando sobre la adaptación de la población infantil a los cambios en los hogares tanto en los inicios de la cuarentena como los cambios presentados una vez iniciados los procesos terapéuticos virtuales y los retos que ha representado para padres, para pacientes y profesionales de la salud mental.

\section{Cotidianidad y Rutinas}

Más allá de los cambios sociales y laborales que trajo consigo el Covid-19, debe agregarse aquí que la situación de cuarentena también dejó expuesto el cambio en las dinámicas familiares. Dado que, las rutinas y los horarios se alteraron considerablemente, de ahí que incluso en algunos hogares se acentuó la inexistencia de rutinas, afectando la salud mental tanto de adultos como de niños, puesto que, al no poder salir de sus hogares o no continuar con labores académicas y laborales también ha implicado que la actividad de los procesos cognitivos en la cotidianidad disminuya, ya que muchas áreas como la alimentación y el sueño que son claves para el aprendizaje (Uscategui, 2016), no se están dando con el mismo cuidado.

Frente a lo anterior, es importante tener claridad de los mecanismos cerebrales que intervienen en los procesos de aprendizaje dependen en gran parte no solo de la genética, sino del entorno y de las emociones, por lo que las instituciones educativas, aunque inicialmente pararon sus labores, entraron en fase de adaptación para las clases virtuales, en un intento por responder a esas demandas actuales permitiendo optimizar, afianzar y mantener la actividad de los procesos cognitivos (Uscategui, 2016), retomando así las jornadas académicas.

Es así, como la importancia de establecer un orden en medio de los cambios que se han enfrentado por la cuarentena, resulta imperativo, especialmente para los niños; precisamente algunos autores desde diversas áreas: salud, educación, social, entre otras, han generado guías y protocolos proporcionando a los padres de familia, un apoyo, aportando recomendaciones específicas que pueden facilitar y ayudar a mantener un equilibrio emocional en los niños logrando hacer diferencias entre el tiempo laboral, el tiempo de ocio y el tiempo en familia (Berasategi, Idoiaga, Dosil, Eiguren, Pikatza \& Ozamiz, 2019).

El sueño es otra de los focos en lo que se ha hecho recomendaciones, donde se hace evidente el mantenimiento de hábitos saludables que permitan las horas de sueño adecuadas, la alimentación por su parte requiere de gran cuidado, pues la ingesta de comida ha incrementado durante la cuarentena, sin embargo, debe tenerse un equilibrio en aquella comida que se le está suministrando a los niños, así mismo, el ejercicio físico y el compartir momentos de esparcimiento con amigos han demostrado una gran importancia para el ser humano durante el confinamiento al no poder realizarse de la forma en 
que se estaba acostumbrado, pues las relaciones e interacciones sociales tuvieron un cambio drástico y aunque muchas de las plataformas on-line ofrecen a los niños la oportunidad de poder ver a sus amigos, es necesario reforzar dichos lazos sociales como parte del fortalecimiento de la salud mental (Berasategi, et. al. 2019).

En este aspecto la Organización Mundial de la Salud (OMS) (2019), ha generado gran cantidad de estrategias donde hace referencia a la importancia de mantener rutinas en casa, la necesidad de establecer tiempos específicos para la alimentación, el sueño, el ocio y claramente incluir dentro de las misma "nueva cotidianidad" rutinas que impliquen en los niños las jornadas académicas, manteniendo sus procesos de aprendizaje.

\section{Cambios emocionales y conductuales}

Frente a los cambios originados por la situación de cuarentena, tanto padres como niños han tenido que adaptarse, intentado llevar una convivencia no solo sana, sino creativa y con varias actividades que permitan el pasar del tiempo más agradable, especialmente en lo referente a la esfera social, pues en el caso de los niños, el no poder socializar ha implicado la aparición de cambios de humor, así como de comportamientos y hasta episodios de ansiedad, algunos autores en España refieren que por el confinamiento actual un $36.23 \%$ de los niños se enfadan algo más de lo habitual, un 26.10\% bastante más de lo habitual y un $12.33 \%$ mucho más, siendo un $74.66 \%$ el que dice que se enfadan más de lo habitual (Berasategi, Idoiaga, Dosil, Eiguren, Pikatza \& Ozamiz, 2019), y aunque en ese mismo estudio, se muestra que un $25.33 \%$ dice que sus hijos/as no se enfadaban más de lo habitual, los porcentajes que presentan frente a la cantidad de niños que si se ven alterados por la situación son superiores.

Adicional a ello, las emociones pueden no seguir un hilo conductor, siendo cambiantes en un mismo día y aunque estén recibiendo clases virtuales y realizando sus deberes académicos, es claro que las condiciones no son las mismas, por ende, se muestra que el $55.54 \%$ de los niños y niñas lloran más, en un $70.17 \%$ están más nerviosos, un $74.66 \%$ tienden a enfadarse más y están más tristes un 55.83\% (Berasategi, et. al., 2019), siendo aquí evidente la importancia de los procesos de socialización e interacción con el entorno para el ser humano, especialmente en sus primeros años, clave en el desarrollo y crecimiento.

Debido a dichos cambios emocionales, no solo se afecta la convivencia en casa, sino que se generan una predisposición al aprendizaje, ya que los procesos cognitivos de los niños se verán influenciados por sus emociones generando poca receptividad, pues se ha evidenciado que altos niveles de estrés provoca que los lóbulos prefrontales se vean alterados dando como resultado que las funciones ejecutivas se afecten considerablemente en su praxis (Uscategui, 2016).

De acuerdo a lo anterior, el mundo entero ha comenzado a reconocer la importancia del mantenimiento de una buena salud mental, de ahí que la Organización Mundial de la Salud (2019) ha generado gran cantidad de estrategias con el fin de mitigar los efectos negativos de la situación actual, así mismo cada país ha establecido protocolos de acción a través de leyes y decretos, tanto en Colombia el Ministerio de Salud y Protección Social (2019) como en Costa Rica el Ministerio de Salud (2019), han entregado algunas recomendaciones para mantener el bienestar emocional y psicosocial de los ciudadanos durante el brote del virus, dentro de ellas se han establecido diferentes estrategias especialmente para los niños.

\section{Niños y asistencia psicológica}

Ahora bien, se ha hecho hincapié en que es la población infantil una de las que más ha recibido los efectos colaterales que ha dejado el Covid-19 en la 
esfera emocional y comportamental, sin embargo, se ha estado hablado de niños que no poseen o que no reciben un proceso terapéutico, entendiendo aquí, que aquellos niños que poseen un diagnóstico podrían estar teniendo más inconvenientes a la hora de asimilar el confinamiento, precisamente algunos autores refieren que en una situación de aislamiento social, niños con Trastorno Déficit de Atención con Hiperactividad (TDAH) son más vulnerables, puesto que eventualmente los síntomas podrían acentuarse y hacer el manejo en casa más complejo, al no tener los padres la presencia de profesionales (Guanilo, Choquicondo, Chamorro \& Florentino, 2019).

Es así que, los procesos de teletrabajo, la teleasistencia, telemedicina, teleorientación o sesiones virtuales se han convertido en una alternativa que en diferentes momentos ha mostrado su efectividad y viabilidad (Spencer, Noyes \& Biederman, 2019), e incluso algunos Centros reconocidos por sus procesos de rehabilitación a través plataformas online como NeuronUP en España, que ofrece servicios a profesionales que atienden a población diversa, tanto infantil como adolescente con diagnósticos de trastornos de retraso en el desarrollo, dificultades de lenguaje, niños con Trastornos del Espectro Autista (TEA), niños con TDAH, niños con alteraciones conductuales o emocionales. han tenido que reorganizar los objetivos de cada uno de sus programas de intervención, adaptándoles a sesiones virtuales (NeuronUP, 2019).

Así mismo, en Latinoamérica algunos entes de salud viendo las necesidades de sus pacientes y siguiendo los lineamientos provisionales para la atención en salud de niñas, niños y adolescentes en el contexto de la pandemia por covid-19 (Ministerio de Salud y Protección Social, 2019 - Colombia, Ministerio de Salud 2019 - Costa Rica), han implementado el teletrabajo como alternativa, con la intención de dar no solo continuidad a los procesos terapéuticos, sino poder garantizar atención de calidad a los pacientes, de ahí que los profesionales se han visto en la tarea de crear y plantear nuevas formas de realizar intervención donde puedan brindar estrategias y técnicas a través de dichas sesiones virtuales.

De acuerdo a ello, profesionales de la salud que atienden a niños que por sus características o diagnósticos requieren de procesos continuos desde de diferentes áreas como física, ocupacional, psicología, neuropsicología y de lenguaje, se han visto en la tarea de "improvisar", a través de la creación de protocolos de intervención que puedan adaptarse a las condiciones y contextos propios de cada casa, de ahí que deben diseñarse directrices frente a las funciones y roles de los participantes, ya que más allá de terapeuta - paciente, también se hace necesario contar con la presencia de padres o cuidadores de los niños (Guanilo, Fernández, Chamorro \& Florentino, 2019), por lo que los entes de salud que cubren dichos servicios han debido coordinar con sus profesionales reuniones online para poder trabajar de manera interdisciplinar y que los tratamientos puedan llevarse a cabo con la mayor calidad posible (NeuronUP, 2019).

No obstante, es importante mencionar aquí que, así como para los profesionales ha representado un reto esta modalidad virtual, para los padres también lo ha sido, dado que ellos pasaron a convertirse en los terapeutas, pues ha de entenderse que por el contexto, los niños requieren de supervisión y de alguien que pueda estar dirigiendo las actividades propuestas por el profesional, de igual manera, algunas sesiones se hacen de forma grupal con la intención de mantener cierto grado de interacción entre los niños, sin embargo, de acuerdo a sus características se organizan dichas sesiones teniendo en cuenta las edades (Guanilo, Choquicondo, Chamorro \& Florentino, 2019).

\section{Metodología e Instrumentos}

La metodología es cuantitativa, de tipo cuasiexperimental (Ato, López, \& Benavente, 2013), dirigida a una población que oscila entre los 0 
y 25 años con algún diagnóstico que involucra discapacidad cognitiva, autismo o trastorno del desarrollo. Es importante mencionar que para el desarrollo de esta investigación y la selección de la muestra se trabajó de forma simultánea en dos países Costa Rica y Colombia, desde la articulación con psicólogos que en medio de la cuarentena retomaron sus procesos terapéuticos a través de las sesiones virtuales, es necesario aclarar que algunas de las sesiones se han realizado de forma grupal y otras de forma individual, por lo que se tomó una muestra de 50 pacientes.

Es así que, de acuerdo a las necesidades de la investigación se creó un cuestionario dividido en dos partes con respuesta en escala Likert, la primera parte hace referencia a cinco reactivos que intentan ahondar sobre los efectos evidenciados en las primeras semanas de la cuarentena donde todavía no se había retomado el proceso psicoterapéutico, la segunda parte del cuestionario consta de cinco reactivos que se enfocarán al impacto obtenido una vez iniciadas las sesiones virtuales.

\section{Resultados y Discusión}

Una vez iniciada la fase de recolección de datos, se confirma el primer aspecto base sobre el cual gira la investigación y es que muchos profesionales y entes de salud, viendo la necesidad de sus pacientes, especialmente en aquellos niños que tienen un diagnóstico y requieren de un proceso terapéutico continuo, retomaron sus tratamientos a través de la virtualidad como alternativa más viable y desde la adaptación de protocolos para la calidad de las mismas (Gráfica 1) (Spencer, Noyes \& Biederman, 2019).

Dentro de lo que puede observarse en la gráfica 1 , muchos de los profesionales de la salud mental están realizando sesiones virtuales en su mayoría con un $34 \%$ a niños con síndrome de Down, 24\% niños con Asperger, 28\% con TDAH, 8\% con Discapacidad Cognitiva, es importante mencionar que el $6 \%$ de los niños que corresponde a diagnósticos sin definir, es precisamente por la edad del mismo, dado que algunos de ellos tienen menos de 5 años y por ende no cumplen aún con una sintomatología específica, sin embargo, presentan alteraciones cognitivas en relación a procesos de desarrollo y por lo cual se encuentra en un proceso terapéutico.

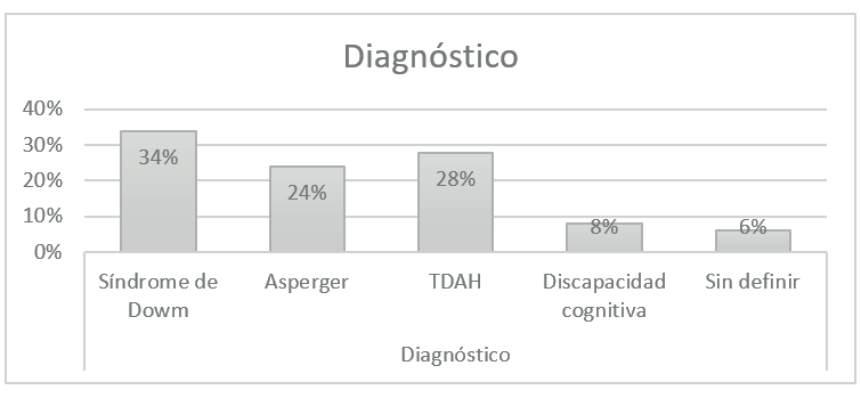

Grafica 1. Diagnóstico de los niños

Dentro de los datos sociodemográficos y que permiten establecer el contexto propio de cada niño se encontró que un 58\% vive con padre, madre y hermanos, un $24 \%$ vive con sus padres biológicos, $12 \%$ solo vive con la madre, $4 \%$ vive con padre y madre siendo uno de ellos padrastro o madrastra y un $2 \%$ equivalente a un solo niño que refiere vive con los abuelos (Gráfica 2), sin embargo, uno de los aspectos sobre los que debe hacerse énfasis, es que una vez iniciadas las sesiones virtuales, de las cifras ya mostradas, el $98 \%$ asiste a las sesiones terapéuticas con la madre y el $2 \%$ que es el paciente que vive con los abuelos, asiste a las sesiones con la abuela.

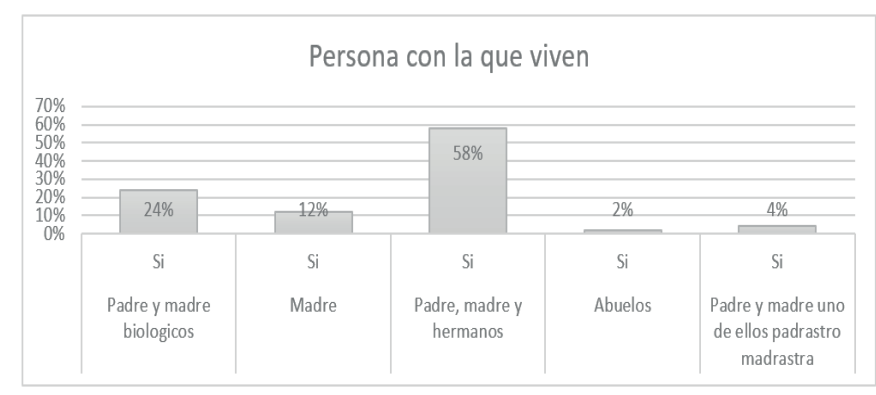

Grafica 2. Persona con la que viven los niños

Ahora bien, el cuestionario se dividió en dos momentos, el primero hacía referencia al impacto de la cuarentena que presentaron los niños durante 
el inicio de la misma y el segundo, al impacto generado una vez iniciadas las sesiones virtuales, de ahí que se indagó por si se vieron afectados negativamente por el confinamiento, encontrando que un $46 \%$ refiere que Totalmente de acuerdo, $40 \%$ De acuerdo y un $14 \%$ de los padres responden la opción de Indeciso, es importante aclarar que para esta última cifra, los niños son menores a 5 años, y el no estar escolarizados ni contar con una rutina extensa, puede haber generado que los efectos negativos no se hayan acentuado del todo.

Así mismo, se indagó por las áreas en las que la cuarentena ha tenido mayor impacto, aquí debe mencionarse que los padres podían elegir varias opciones por lo que como totalidad no daría un $100 \%$, ya que precisamente la intención del ítem correspondía en identificar si existían varias áreas afectadas y cuales eran. De acuerdo a ello, se encontró que la mayor área fue la emocional con un $98 \%$, el área de comportamiento con un $88 \%$ y sueño con un $62 \%$, el área de alimentación no se vio alterada (Gráfica 3). Aquí ha de relacionarse un ítem que se planteó sobre si dejar de hacer actividad física ha afectado o no a los niños, frente a ello, los padres refieren en un $100 \%$ estar totalmente de acuerdo, lo que explica también el impacto negativo en las áreas ya mencionadas. Lo anterior respalda lo expresado con algunos autores en España quienes mencionaban que por el confinamiento muchos niños se enfadaban, lloraban, y estaban más tristes y nerviosos de lo habitual, entendiendo que los cambios dentro de la cotidianidad alteran considerablemente la estabilidad y salud mental de la población infantil (Berasategi, et. al., 2019).

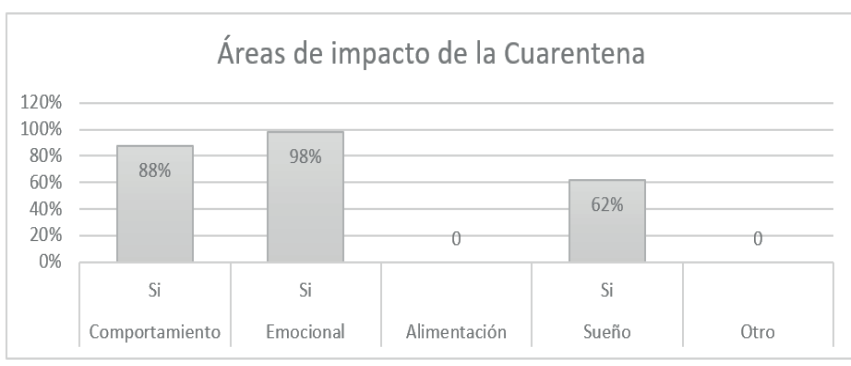

Grafica 3. Áreas de impacto de la Cuarentena

Aquí se abre espacio para las comparaciones y es que dentro del cuestionario se indagó si después de iniciadas las sesiones virtuales, se presentó o evidenciaron algún impacto positivo, encontrando aquí que el $100 \%$ de los padres respondieron Totalmente de acuerdo, siendo así que profundizó nuevamente en las áreas por las que se tuvo mayor impacto, e igualmente en este ítem, también podía seleccionarse varias áreas. De acuerdo a ello, se encontró que las sesiones tuvieron un efecto positivo en las áreas emocional con un $98 \%$ y comportamental un $72 \%$ (Gráfica 4), siendo afín a lo expresando por NeuronUP (2019), pues dentro de los resultados encontrados en las adaptaciones de sus protocolos a la virtualidad, refieren que se ha evidenciado una evolución en aquellos niños que reciben sus terapias virtuales, frente aquellos que no.

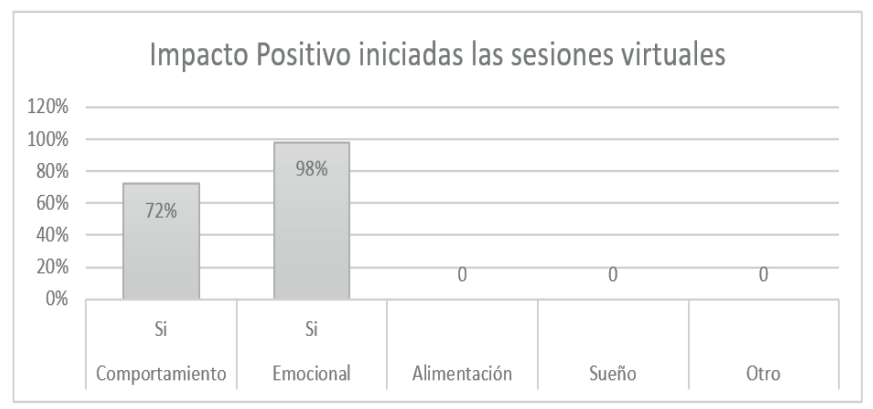

Grafica 4. Impacto positivo iniciadas las sesiones virtuales

Es así que siguiendo con la información anterior, también se quiso profundizar por la importancia de las interacciones sociales, en donde se preguntó en el primer momento si los padres consideran que a los niños les afectó negativamente dejar de ver a sus compañeros y amigos, un 94\% refiere estar Totalmente de acuerdo y un $6 \%$ está De acuerdo 
con el ítem, así mismo, en el segundo momento se efectuó el mismo ítem, indagando si afectó positivamente el volver a ver a sus compañeros y amigos, un $70 \%$ refiere estar Totalmente de acuerdo y un $30 \%$ De acuerdo. Para estas últimas cifras es importante mencionar que las clases virtuales ya se hayan iniciado e igualmente muchas de las sesiones se realizaban tanto individual como grupal, lo que podría explicar una mejora en el área emocional como se muestra en la gráfica 4 y que haya impactado positivamente en diferente grado en los pacientes, y es que precisamente dentro de las recomendaciones que se dieron por parte de la OMS (2019) y autores como Berasategi, et. al. (2019), se señala la importancia de mantener las relaciones e interacciones sociales como parte del fortalecimiento de la salud mental en los niños.

Ahora bien, los cambios que trajo consigo el covid-19 implicó la perdida de rutinas, ya que trabajos e instituciones educativas pararon sus labores, precisamente se indagó por la cotidianidad al interior de los hogares, donde se encontró que el 100\% de los participantes refieren que durante el inicio de la cuarentena no contaban con rutinas establecidas en casa, sin embargo, una vez iniciadas las sesiones terapéuticas, para el mismo ítem, el 100\% de los participantes expresan que si cuentan actualmente con rutinas, frente a ello, debe mencionarse que para ese momento, muchas instituciones educativas ya habían iniciado su procesos de clases virtuales, así mismo, los profesionales de la salud que participaron en la investigación manifiestan que una de las sugerencias que dieron al comienzo de las sesiones fue el retomar y/o crear rutinas en casa con la intención de mantener la salud mental de los niños, recomendaciones que también establecen la OMS (2019) y autores como Berasategi, et. al. (2019) como parte del proceso de adaptación al confinamiento.

Teniendo en cuenta que por el contexto que se establece en la virtualidad, hace necesaria la presencia de los padres en las sesiones para que puedan dirigir y supervisar las actividades, se creó un ítem, con el fin de saber si asumir el rol de ser terapeutas en casa ha representado un reto para ellos, a lo que el $100 \%$ de los padres refieren estar Totalmente de acuerdo con ello, entendiendo que las demandas en casa aumentan no solo con los cuidados propios de los hijos sino también como cuidadores -terapeutas durante las sesiones virtuales.

\section{Conclusiones}

La presente investigación deja en evidencia no solo los impactos propios del confinamiento por el covid-19 sino las dinámicas familiares alrededor de niños que cuentan con un proceso terapéutico debido a un diagnóstico, de lo anterior es clara la importancia del mantenimiento de rutinas dentro de la cotidianidad, ya que ayuda a tener un equilibrio y tiempos establecidos para cada actividad, generando diferencias y evitando sobrecarga de trabajo.

Así mismo, la información suministrada expone, que es la población infantil no solo una de las más afectadas, sino que de forma colateral ha dejado consecuencias especialmente en las áreas emocional, comportamental y de sueño, siendo importante aquí crear estrategias al interior de los hogares que permitan mitigar dichos efectos negativos, puesto que se evidenció un cambio considerable posterior al inicio de las sesiones virtuales donde los niños lograron recobrar un poco de la cotidianidad que tenían anteriormente, obteniendo un impacto positivo no solo por el mantenimiento de las interacciones sociales que ofrece la virtualidad sino por el hecho de retomar los objetivos de cada uno de los procesos terapéuticos que tenían los niños, a través de diferentes plataformas con la intención de mantener un tratamiento que permita evolución de sus procesos cognitivos evitando un estancamiento o dependiendo del caso, perdida de habilidades que todavía no estaban consolidadas (NeuronUP, 2019).

Ahora bien, es claro que uno de los aspectos sobre el cual quedaron inquietudes y por lo que se 
deja abierta la puerta para futuras investigaciones, es que es la madre la que hace frente a los procesos terapéuticos de los niños con algún diagnóstico, aun viviendo con otras personas, así mismo, se abre nuevas líneas de investigación frente al apoyo que se recibe en casa, tanto los mismos niños como sus cuidadores que en este caso son las madres, siendo cuestionable si es un suceso propio de la cuarentena o en la cotidianidad se presenta la misma situación.

\section{Referencias}

Ato, M., López, J. J., \& Benavente, A. (2013). Un sistema de clasificación de los diseños de investigación en psicología. Anales de psicología, 29(3), 1038-1059.

Berasategi Sancho, N., Idoiaga Mondragón, N., Dosil Santamaría, M., Eiguren Munitis, A., Pikatza Gorrotxategi, N., \& Ozamiz Echevarria, N. (2019). Las voces de los niños y de las niñas en situación de confinamiento por el COVID-19. Servicio de Publicaciones de la Universidad del País Vasco.

Guanilo, C. D. A., Fernández, T. B. L., Chamorro, A. S. T., \& Florentino, B. S. V. (2019). Teleasistencia psicológica para niños con Trastorno del Espectro Autista: definiciones, directrices y tendencias tecnológicas para el trabajo basado en evidencia. Instituto Psicopedagógico EOS Perú.

Guanilo, C. D. A., Choquicondo, C. M., Chamorro, A. S. T., \& Florentino, B. S. V. (2019). Teleasistencia psicológica para niños en edad escolar con Trastorno por Déficit de Atención/ Hiperactividad: Rol de los padres de familia y cuidadores. Instituto Psicopedagógico EOS Perú.

Ministerio de Salud (2019). Lineamientos Nacionales para la Vigilancia de la infección por Coronavirus (COVID-19). Website de Costa Rica. Recuperado de: https://www. ministeriodesalud.go.cr/index.php/centrode-prensa/noticias/741-noticias-2020/1532lineamientos-nacionales-para-la-vigilancia-dela-infeccion-por-coronavirus-2019-ncov

Ministerio de Salud y Protección Social (2019)
Lineamientos provisionales para la atención en salud de niñas, niños y adolescentes en el contexto de la pandemia por covid-19 en Colombia (2019). Publicado en mayo de 2020. Bogotá - Colombia.

Ministerio de Salud y Protección Social (2019). Todos a cuidar nuestra salud mental durante la COVID-19. Publicado el 28 de marzo de 2019. Boletín de Prensa No 105 de 2019. Colombia. Website de Colombia. Recuperado de: https:// www.minsalud.gov.co/Paginas/Todos-a-cuidarnuestra-salud-mental-durante-la-COVID-19. aspx.

NeuronUP (2019). Aprendiendo con el COVID-19: neuropsicología online. Publicado el 08 de junio de 2019. Tomado el 09 de junio de 2019. Website de España. Recuperado de: https://blog. neuronup.com/neuropsicologia-online/

Organización Mundial de la Salud (2019). Se publica un cuento infantil para ayudar a niños y jóvenes a hacer frente a la pandemia de COVID-19. Publicado el 9 de abril de 2019. Tomado el 9 de junio de 2019. Recuperado de: https://www.who. int/es/news-room/detail/09-04-2019-childrens-story-book-released-to-help-children-andyoung-people-cope-with-covid-19

Spencer, T., Noyes, E., \& Biederman, J. (2019). Telemedicine in themanagement of ADHD: Literature Review of Telemedicine in ADHD. Journal of Attention Disorders, 24 (1). Tomasi, S. N. (2019). Historia De Las Pandemias Mundiales Y La Economía.

Uscategui, M. Boscan, A. (2016). Neuroeducación Un Enfoque Motivador Para el Proceso de Aprendizaje del Cálculo Inicial En La Universidad Francisco de Paula Santander. Encuentro Internacional en Educación Matemática ISSN 2539-1885. La Educación Matemática como Herramienta en el Desempeño Profesional Docente. Cúcuta, Colombia. 16 - 24. 